\title{
Five Month-Persistent Extrapyramidal Symptoms following a Single Injection of Paliperidone Palmitate: A Case Report
}

\author{
Seoyoung Jang ${ }^{1}$, Jungmin Woo ${ }^{2}$ \\ ${ }^{1}$ Department of Psychiatry, Catholic University of Daegu School of Medicine, ${ }^{2}$ Department of Psychiatry, Kyungpook National University School \\ of Medicine, Daegu, Korea
}

\begin{abstract}
Long-acting injectable (LAl) antipsychotics are useful in the treatments for schizophrenic patients with poor adherence due to their maintaining feature of therapeutic plasma level without daily administrations. However, their long-lasting property can cause complicated problems such as a long-lasting side effect. We report a patient who experienced LAl-induced extrapyramidal symptoms (EPSs) for 5 months after a single injection. During that period, every trial to ameliorate this condition turned out to be a failure. The 3-month formulation of paliperidone palmitate is now close at hand. We have to be aware of possible long-lasting adverse events and confirm the tolerability to LAl before use.
\end{abstract}

KEY WORDS: Long-acting injectable antipsychotics; Paliperidone palmitate; Extrapyramidal symptoms; Schizophrenia.

\section{INTRODUCTION}

Paliperidone palmitate is one of long-acting injectable (LAI) antipsychotic drugs used for schizophrenia with poor-adherence. It has a benefit of maintaining therapeutic plasma level with a four-week interval of injections. ${ }^{1,2)}$ Therefore, it can decrease recurrence rate and hospitalization, particularly in the patients with pooradherence. In practice, paliperidone palmitate is used frequently due to its long-acting property. Recent studies have reported its extended availability for acute-phase and first-phase treatment, ${ }^{3,4)}$ and the formulation that maintains therapeutic level longer ( 3 months) has been introduced to clinicians.

However, we sometimes observed unexpected paliperidone palmitate-induced extrapyramidal symptoms (EPSs). One study showed high incidence of paliperidone palmitate-induced EPSs based on the Simpson-Angus Scale (SAS) scored by clinicians (18\% in the $100 \mathrm{mg} /$ day group) ${ }^{5,6)}$ This result suggests LAI-induced EPSs may be actually more frequent but unrecognized and underestimated. Long acting intramuscular formulation is slowly

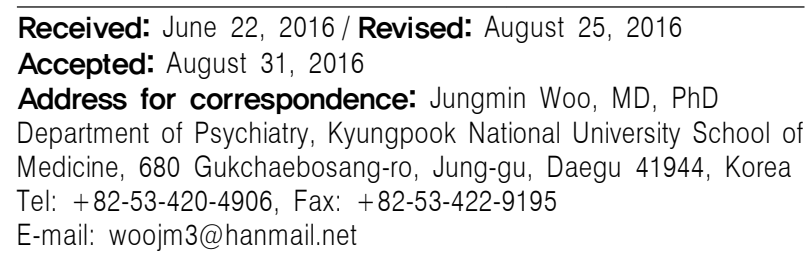

released from injection site for several weeks. If LAI-induced EPSs appear, it may be quite challenging to manage them in clinical fields. We report a case of LAI-induced EPSs lasting for 5 months in a schizophrenia patient and raise a question about safety in standard guidance of LAI antipsychotics. Informed consent was taken from the patient for the publication of this case report.

\section{CASE}

A 53-year-old female, who had been diagnosed with schizophrenia 30 years ago, was referred to our psychiatric clinic for management of uncontrolled psychotic symptoms (delusion, loosening of association) and severe EPSs (tremor, rigidity and gait unstability).

Four months ago, she was admitted to a local psychiatric hospital with relapse of psychotic symptoms. Due to previous history of poor adherence to medication, 156 $\mathrm{mg}$ /day paliperidone palmitate (paliperidone $100 \mathrm{mg} /$ day) was injected on her deltoid muscle. Seven days after first injection, severe EPSs appeared and she frequently fell down due to gait instability. So the scheduled second injection was skipped and all medications were stopped to prevent aggravations of these symptoms. After that, she maintained oral antipsychotics-free state for a month and additional medications were prescribed to reduce EPSs. But LAI-induced EPSs had persisted without improvement.

Despite of the presence of severe EPSs, oral anti-

(a) This is an Open-Access article distributed under the terms of the Creative Commons Attribution Non-Commercial License (http://creativecommons.org/licenses/by-nc/4.0) which permits unrestricted non-commercial use, distribution, and reproduction in any medium, provided the original work is properly cited. 
psychotics were administered twice during 4 months for dealing with her psychotic symptoms. However, each of the trials made her EPSs worse seriously. Only the discontinuation of oral antipsychotics could relieve the severity of EPSs as before adding of oral antipsychotics: one month after the development of EPSs, she was prescribed aripiprazole $5 \mathrm{mg} /$ day. After that, the truncal tremor and gait instability were aggravated rapidly, so aripiprazole was discontinued the very next day. After 3 months, quetiapine was started and gradually titrated up at 25-50 mg every 3-4 days until it reached $150 \mathrm{mg} /$ day. During this period, her tremor worsened and she fell down more frequently. Quetiapine was gradually tapered off over next 5 days. Her prognosis is demonstrated below (Fig. 1).

About 4 months later from a manifestation of severe EPSs, she was admitted to our psychiatric hospital. She had multiple bruises on her face, elbow and knee and couldn't walk alone without assistance. Also she couldn't eat anything by herself due to her shaking hands. She exhibited hand tremor, rigidity, bradykinesia and postural instability. Her EPSs were evaluated with total 17 points in SAS (gait, 3/4; arm dropping, 3/4; shoulder shaking, $1 / 4$; elbow rigidity, $2 / 4$; leg pendulousness, $1 / 4$; head dropping, 2/4; tremor, 3/4; salivation, 2/4). Brain magnetic resonance imaging study, electroencephalography and laboratory tests did not reveal any significant findings.

We had observed her problems without any antipsychotics for one month. During that period, we prescribed procyclidine (10 mg/day), propranolol (40 mg/day), lorazepam ( $2 \mathrm{mg}$ /day) and flunitrazepam ( $1 \mathrm{mg}$ /day) for the purpose of reducing EPSs and mitigating psychotic symptoms.

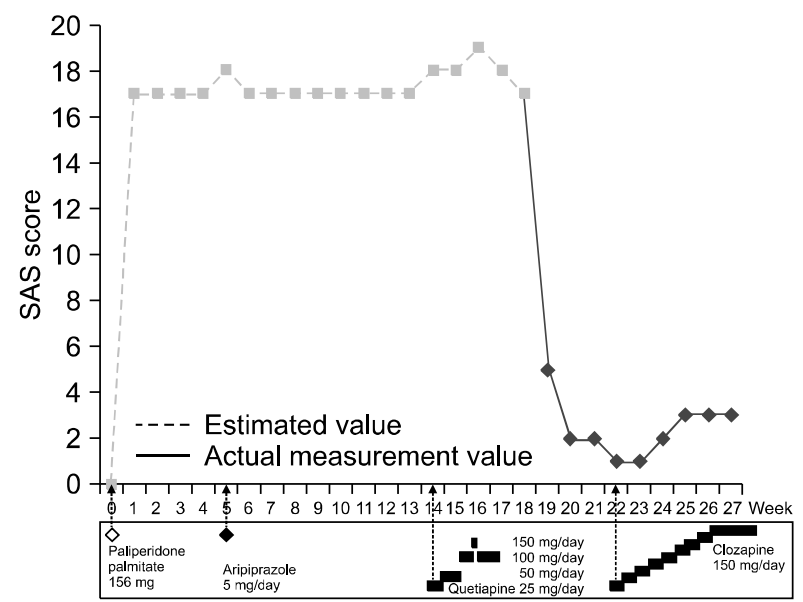

Fig. 1. Course of the Simpson-Angus Scale (SAS) score.
As full remission of EPSs did not show after enough long time for total excretion of paliperidone palmitate from her body, we decided to start titration of clozapine carefully. During one month, the dose of clozapine was increased to $150 \mathrm{mg}$. We observed her psychotic symptoms, including loosening of association and delusional thoughts and her scoring on the Positive and Negative Syndrome Scale (PANSS) had gradually decreased after the onset of medication (total PANSS score 80, before clozapine medication; 55, one month after medication). Her psychotic symptoms were stabilized and she was discharged 6 months after acute exacerbation. After discharge, she is still under outpatient follow-up and has showed no EPS reoccurrence until now.

\section{DISCUSSION}

Paliperidone palmitate is safe and tolerable. Previous studies showed that schizophrenia treated with paliperidone palmitate showed lower incidence rate of EPSs compared with oral paliparidone. ${ }^{6,7)}$ However, in this case, paliperidone palmitate-induced EPSs had persisted for 5 months after a single injection. Whenever low-dose antipsychotics were added, EPSs showed serious worsening and anticholinergic drugs did nothing for improving EPSs during this period.

EPSs depend on serum concentration of antipsychotics. High-dose antipsychotics cause increased D2 occupancy in striatum and EPSs develop when levels of concentration are over EPS threshold. ${ }^{8)}$ In most cases, the serum level of paliperidone is dropping over time and becomes lower than the therapeutic level one month after injection. ${ }^{9,10}$

There are several hypothesises that may account for high sensitivity which causes long-lasting EPSs secondary to paliperidone palmitate injection.

First, paliperidone palmitate may work in a relatively narrow range of the therapeutic window (D2 occupancy $65-72 \%)$ and at the same time it showed a wide range of D2 occupancy depending on individual factors $(38-87 \%) .{ }^{8)}$ This result means that some individuals in the extreme pole can be highly vulnerable to EPSs. Second, some of the individual factors in terms of pharmacokinetics may have increased serum concentration over the expected level of the maximum plasma concentration (Cmax) and the area under the plasma concentration-time curve (AUC). But there aren't enough studies or evidence to support this hypothesis. Third, D2 binding potential could be related with EPS. In one previous study, patients who experienced clinically relevant EPS showed sig- 
nificantly higher mean striatal D2 receptor occupancy $(77 \%)$ than those without EPS $(61 \%) .{ }^{11)}$ Most antipsychotics compete with endogenous dopamine for striatal D2 receptor. ${ }^{12)}$ Low affinity to D2 receptor of endogenous dopamine is associated with higher striatal D2 receptor occupancy of antipsychotics which causes EPSs. We assume that the individual factors like endogenous dopamine release and affinity may have an influence on neuroleptic sensitivity with respect to developing paliperidone palmitate-induced EPSs.

In addition, paliperidone palmitate may remain in the body for at least 5 months. The half-life of paliperidone palmitate ranges from 25 days to 50 days. ${ }^{13)}$ At the first injection, the half-life is more variable according to dose or injection site. ${ }^{14-16)}$ Like this case, when $156 \mathrm{mg}$ of paliperidone palmitate is given for the first time, the half-life is 40 days or more. And total excretion time is expected to be 125 days to 250 days because total excretion time after one injection is 5 times as long as the half-life. ${ }^{9)}$ This can explain how EPSs persist for 5 months. If the individual has high sensitivity to antipsychotics, severe EPSs can last for quite a long time when low-level antipsychotics continuously release.

Paliperidone is relatively safe and tolerable in relation to EPSs. ${ }^{17)}$ When EPSs occur, it can be controlled with anticholinergic drugs. Even if severe EPSs that cannot be controlled by anticholinergic drugs develop, we may be able to overcome these problems through drug reduction or discontinuation. However, LAI-induced EPSs may be not easily handled in this way. The recent availability of LAI antipsychotic is increasing and 3-month formulation of paliperidone will be at hand. Up to now, we can think of high doses of antipsychotics, history of EPS and comorbidity as risk factors of EPSs.

To avoid unexpected serious side-effects, we have to be careful for the patient vulnerable to induced EPSs before the use of LAI antipsychotics. In this case, the patient had a family history of Parkinsonism and had experienced EPSs with oral formulation of paliperidone 2 years ago. Before injection of LAI-antipsychotic, drug history and tolerability to oral formulation should be taken into account, while physical or neurological examination should be performed thoroughly before and after administration. When the vulnerability of EPSs is identified, the uniform loading dose regimen ${ }^{18)}$ may be no longer safe. Prolonged uncontrolled EPSs make the patient's treatment adherence poor and increases financial burden due to prolonged hospitalization. The clinicians should carefully consider the vulnerability of EPS based on the individual characteristics.

\section{REFERENCES}

1. Ereshefsky L, Saklad SR, Jann MW, Davis CM, Richards A, Seidel DR. Future of depot neuroleptic therapy: pharmacokinetic and pharmacodynamic approaches. J Clin Psychiatry 1984:45:50-59.

2. Brissos S, Veguilla MR, Taylor D, Balanzá-Martinez V. The role of long-acting injectable antipsychotics in schizophrenia: a critical appraisal. Ther Adv Psychopharmacol 2014:4:198-219.

3. Schreiner A, Aadamsoo K, Altamura AC, Franco M, Gorwood P, Neznanov NG, et al. Paliperidone palmitate versus oral antipsychotics in recently diagnosed schizophrenia. Schizophr Res 2015;169:393-399.

4. Li H, Turkoz I, Zhang F. Efficacy and safety of oncemonthly injection of paliperidone palmitate in hospitalized Asian patients with acute exacerbated schizophrenia: an openlabel, prospective, noncomparative study. Neuropsychiatr Dis Treat 2015;12:15-24.

5. Kramer M, Litman R, Hough D, Lane R, Lim P, Liu Y, et al. Paliperidone palmitate, a potential long-acting treatment for patients with schizophrenia. Results of a randomized, double-blind, placebo-controlled efficacy and safety study. Int $J$ Neuropsychopharmacol 2010;13:635-647.

6. Gopal S, Liu Y, Alphs L, Savitz A, Nuamah I, Hough D. Incidence and time course of extrapyramidal symptoms with oral and long-acting injectable paliperidone: a posthoc pooled analysis of seven randomized controlled studies. Neuropsychiatr Dis Treat 2013;9:1381-1392.

7. Adams CE, Fenton MK, Quraishi S, David AS. Systematic meta-review of depot antipsychotic drugs for people with schizophrenia. Br J Psychiatry 2001;179:290-299.

8. Kapur S, Zipursky R, Jones C, Remington G, Houle S. Relationship between dopamine D(2) occupancy, clinical response, and side effects: a double-blind PET study of firstepisode schizophrenia. Am J Psychiatry 2000;157:514-520.

9. Gopal S, Gassmann-Mayer C, Palumbo J, Samtani MN, Shiwach R, Alphs L. Practical guidance for dosing and switching paliperidone palmitate treatment in patients with schizophrenia. Curr Med Res Opin 2010;26:377-387.

10. Samtani MN, Vermeulen A, Stuyckens K. Population pharmacokinetics of intramuscular paliperidone palmitate in patients with schizophrenia: a novel once-monthly, longacting formulation of an atypical antipsychotic. Clin Pharmacokinet 2009;48:585-600.

11. Tauscher J, Küfferle B, Asenbaum S, Tauscher-Wisniewski S, Kasper S. Striatal dopamine-2 receptor occupancy as measured with [123I]iodobenzamide and SPECT predicted the occurrence of EPS in patients treated with atypical antipsychotics and haloperidol. Psychopharmacology (Berl) 2002;162:42-49.

12. Seeman P, Tallerico T. Antipsychotic drugs which elicit little or no parkinsonism bind more loosely than dopamine to brain D2 receptors, yet occupy high levels of these receptors. Mol Psychiatry 1998;3:123-134.

13. Owen RT. Paliperidone palmitate injection: Its efficacy, safety and tolerability in schizophrenia. Drugs Today (Barc) 2010;46:463-471.

14. Si T, Su Y, Liu Y, Zhang H, Li H, Rui Q, et al. Pharmacokinetics and tolerability of paliperidone palmitate injection in Chinese subjects. Hum Psychopharmacol 2014;29: 203-210.

15. Cleton A, Rossenu S, Crauwels H, Berwaerts J, Hough D, Gopal S, et al. A single-dose, open-label, parallel, randomized, dose-proportionality study of paliperidone after intra- 
muscular injections of paliperidone palmitate in the deltoid or gluteal muscle in patients with schizophrenia. J Clin Pharmacol 2014;54:1048-1057.

16. Ravenstijn P, Remmerie B, Savitz A, Samtani MN, Nuamah I, Chang CT, et al. Pharmacokinetics, safety, and tolerability of paliperidone palmitate 3-month formulation in patients with schizophrenia: A phase-1, single-dose, randomized, open-label study. J Clin Pharmacol 2016;56:330-339.

17. Kang NI, Koo BH, Kim SW, Kim JH, Nam B, Lee BJ, et al. Efficacy and tolerability of paliperidone extended-release in the treatment of first-episode psychosis: An eight-week, open-label, multicenter trial. Clin Psychopharmacol Neurosci 2016;14:261-269.

18. González-Rodríguez A, Catalán R, Penadés R, Garcia-Rizo C, Bioque M, Parellada E, et al. Profile of paliperidone palmitate once-monthly long-acting injectable in the management of schizophrenia: long-term safety, efficacy, and patient acceptability - a review. Patient Prefer Adherence 2015;9:695-706. 